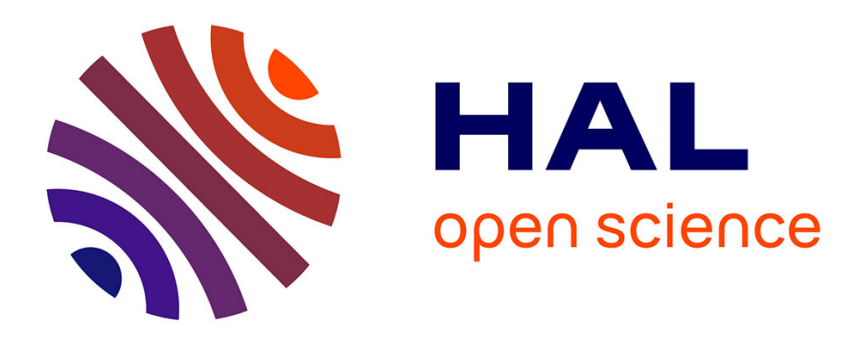

\title{
Stéréotypage et artifice documentaires
}

Caroline Courbières

\section{To cite this version:}

Caroline Courbières. Stéréotypage et artifice documentaires. Communication \& langages, 2011, 170, pp.97-106. 10.4074/S0336150011014086 . hal-01228526

\section{HAL Id: hal-01228526 \\ https://hal.science/hal-01228526}

Submitted on 13 Nov 2015

HAL is a multi-disciplinary open access archive for the deposit and dissemination of scientific research documents, whether they are published or not. The documents may come from teaching and research institutions in France or abroad, or from public or private research centers.
L'archive ouverte pluridisciplinaire HAL, est destinée au dépôt et à la diffusion de documents scientifiques de niveau recherche, publiés ou non, émanant des établissements d'enseignement et de recherche français ou étrangers, des laboratoires publics ou privés. 


\section{Communication \& langages}

http://www.necplus.eu/CML

Additional services for Communication \&

langages:

Email alerts: Click here

Subscriptions: $\underline{\text { Click here }}$

Commercial reprints: $\underline{\text { Click here }}$

Terms of use : $\underline{\text { Click here }}$

\section{Stéréotypage et artifice documentaires}

\section{Caroline Courbières}

Communication \& langages / Volume 2011 / Issue 170 / December 2011, pp 97 - 106

DOI: 10.4074/S0336150011014086, Published online: 02 January 2012

Link to this article: http://www.necplus.eu/abstract_S0336150011014086

How to cite this article:

Caroline Courbières (2011). Stéréotypage et artifice documentaires. Communication \& langages, 2011, pp 97-106 doi:10.4074/S0336150011014086

Request Permissions : $\underline{\text { Click here }}$ 


\section{Stéréotypage et artifice documentaires}

Terme de typographie à l'origine ${ }^{1}$, le stéréotype a acquis au cours du $\mathrm{XX}^{\mathrm{e}}$ siècle un statut conceptuel, devenant l'objet de théorisations distinctes dans divers champs scientifiques. Proche du cliché, il nous entraîne irrémédiablement "vers la forme canoniale, contraignante du signifié $»^{2}$. Le stéréotype s'impose sous la forme d'une représentation langagière qui momifie le signe, à l'image d'une persistance rétinienne négative forcément fatale. L'analyse du mécanisme qui le produit permet de montrer ses masques et ses modes d'apparition, soulignant le caractère perpétuel ou intermittent de sa charge idéologique. Repère ou écueil des mémoires, le stéréotype retourne le simulacre.

Cette figure de second niveau ${ }^{3}$ marque ainsi le point d'équilibre de la rhétorique de la mode ${ }^{4}$ : l'étude de son discours explique comment s'élaborent, conjointement aux signes de mode produits, des représentations féminines figées, sitôt projetées dans l'espace social ${ }^{5}$. La mode vestimentaire, à travers le discours médiatique qui l'instaure, s'avère être un terrain de prédilection pour

1. Alain Rey, Dictionnaire historique de la langue française, Le Robert, Paris, 1998, p. 3640.

2. Roland Barthes, Le plaisir du texte, Éditions du Seuil, Paris, 1973, p. 69.

3. Georges Molinié, Éléments de stylistique française, PUF, Paris, 1986.

4. Roland Barthes, Système de la mode, Éditions du Seuil, Paris, 1967.

5. Caroline Courbières, «Vers une typologie de l'information médiatique au travers de l'analyse des discours sur la mode », Communication \& langages, 134, décembre 2002, pp. 84-97.

\section{CAROLINE COURBIÈRES}

Les langages documentaires, outils professionnels de condensation de l'information, se présentent comme des systèmes de représentation des connaissances. Ils sont ici étudiés comme des discours produisant leur propre système de représentations et générant un processus de stéréotypage singulier. Ce stéréotypage, qui s'appuie sur la notion d'artifice documentaire, dépend des trois types de relations sémantiques qui fabriquent le contexte de signification des unités documentaires. Sa première étape réside dans le principe de figement documentaire selon lequel la signification d'une unité se trouve stabilisée par les relations d'équivalence qui lui octroient son statut documentaire, puis figée par les relations hiérarchiques qui l'inscrivent dans un cadre thématique identifié. Sa seconde étape relève du phénomène de fragmentation documentaire réalisé par les relations associatives qui projettent le signifié de l'unité dans un (ou plusieurs) autre(s) domaine(s) que sa classe d'inscription première. 
l'étude des stéréotypes, mais d'autres sphères discursives peuvent être abordées à l'aune du principe de stéréotypage.

Les langages documentaires utilisés par les professionnels de l'information (documentalistes, bibliothécaires) pour traiter leurs ressources peuvent également être appréhendés comme lieux de construction de représentations sociales de référence ${ }^{6}$. Ces systèmes langagiers servent à représenter de manière condensée sous forme de signes (descripteurs, indices, etc.) - le contenu informationnel des documents afin d'en faciliter à la fois l'accès et l'exploitation. Bien que la comparaison puisse surprendre - ou que le terrain paraisse moins glamour -, on peut pourtant faire un parallèle entre le discours sur la mode délivré par les instances spécialisées et le discours documentaire produit par ces langages dits artificiels composés des thésaurus, classifications et autres ontologies.

Systèmes de représentation du mondain documentaire, ces langages sont non seulement des produits idéologiques par leur caractère descriptif, mais aussi des producteurs d'idéologies par leur caractère normatif. En tant qu'outils documentaires, ils se basent sur la représentation du monde inhérente à la masse documentaire qu'ils doivent décrire et présentent un caractère de légitimation à double détente : les langages documentaires servent tout à la fois à légitimer certaines pratiques qui cimentent la cohésion d'un groupe professionnel et à renforcer l'influence que peut avoir ce groupe social sur le plus grand nombre. Il convient dès lors d'analyser selon quelles modalités ces outils de représentation des connaissances génèrent leur propre processus de stéréotypage.

\section{LE MONSTRE SOUS LES MOTS}

La notion de stéréotype - à la fois commune et spécialisée, selon ses lieux d'application - est depuis longtemps travaillée par la psychologie sociale, qui vise notamment à détailler le processus cognitif de stéréotypisation et le rôle des stéréotypes dans le traitement des informations ${ }^{7}$; elle occupe aussi une place centrale en sémantique du stéréotype et du prototype. Dans la lignée de la conception aristotélicienne selon laquelle la catégorisation s'opère sur la base de propriétés communes et suite à la théorie sémantique du philosophe Hilary Putnam sur les noms des espèces naturelles - pour lesquels la signification des lexèmes renvoie aux traits descriptifs de leurs référents -, le stéréotype est ce qui caractérise un membre normal de l'espèce ${ }^{8}$. La notion sert également d'outil opérationnel pour définir la relation entre une unité et son référent extralinguistique : cette sémantique du prototype, qui rejoint la théorie de la

6. Bernard Py, « Pour une approche linguistique des représentations sociales », Langages, 154, juin 2004, pp. 6-19.

7. Jean-Philippe Leyens, Vincent Yzerbit et Georges Schadron, Stéréotypes et cognition sociale, Mardaga, Liège, 1996; Vincent Yzerbit, Georges Schadron, « Stéréotypes et jugement social » in Richard Y. Bourhis et Jean-Philippe Leyens, Stéréotypes, discrimination et relations intergroupes, Mardaga, Liège, 1999, pp. 127-160.

8. Cf. Ruth Amossy et Anne Herschberg Pierrot, Stéréotypes et clichés : Langue, discours, société, Nathan, Paris, 1997. 
psychologue Eleanor Rosch, définit le prototype comme le meilleur exemplaire communément associé à une catégorie et le stéréotype comme son concept : « Les deux se rejoignent dans le cas standard, dans la mesure où les données sémantiques les plus importantes d'un point de vue social sont aussi celles qui sont les plus importantes dans l'organisation cognitive des catégories $»{ }^{9}$.

Pour Walter Lippmann, qui développe le premier cette notion en sciences sociales, le stéréotype est une image qui médiatise notre rapport au monde : "In the great blooming, buzzing confusion of the outer world we pick out what our culture has already defined for us, and we tend to perceive that which we have picked out in the form stereotyped for us by culture ${ }^{10}$. Grâce au stéréotype, le sens que l'on construit des choses se trouve à la fois précisé et solidifié. Dans le prolongement de cette idée, le stéréotype est considéré comme une grille de lecture qui fige une image simple à l'attention de la communauté ${ }^{11}$. Pour notre part, la notion de stéréotype est envisagée comme un point de fuite où convergent la thématique de la construction des connaissances et les études culturelles. Cette voie d'investigation, et l'approche sémio-communicationnelle adoptée, vise à saisir le processus de cristallisation langagière selon lequel une unité informationnelle (c'est-à-dire un élément relevant du système de la langue ou d'autres systèmes langagiers) voit se solidifier sa forme de l'expression - éventuellement soumise à variation - associée à un contenu stable. Poser le principe de stéréotypage autorise à déconstruire cette association et à spécifier le phénomène de naturalisation à l'œuvre dans le processus de production/interprétation. Cet angle d'attaque permet de comprendre comment et pourquoi cette représentation signifie dans des horizons déterminés.

L'examen de la mode vestimentaire, indissociable de sa médiatisation, montre par exemple comment les éléments informationnels et communicationnels de son discours favorisent la construction de représentations féminines stéréotypées, constituant ce que le photographe Peter Lindbergh appelle « la propagande de la femme ». Cette construction autour de l'information de mode s'opère selon trois processus distincts : soit la figure féminine va s'élaborer à partir de l'actualisation d'un mot clé, soit elle va s'ancrer dans un contexte culturel déterminé, soit, enfin, elle va permettre la stabilisation de cadres de référence qui participe de la reconstruction d'un certain imaginaire spatial ${ }^{12}$. C'est ainsi que certaines figures de la femme se trouvent cristallisées par l'actualisation de cadres fortement identifiables. Ce faisant, le discours sur la mode construit son propre système

\section{Georges Kleiber, La sémantique du prototype : Catégories et sens lexical, PUF, Paris, p. 69.}

10. «Dans l'immense et active confusion du monde extérieur, nous sélectionnons ce que notre culture a déjà défini pour nous, et nous avons tendance à percevoir ce que la culture a sélectionné pour nous sous la forme stéréotypée. » Walter Lippmann, Public Opinion, Penguin Books, New York, [1922] 1946. Texte disponible [en ligne] : adresse URL : http://xroads.virginia.edu/ Hyper2/CDFinal/Lippmann.html

11. Ruth Amossy, Les idées reçues : Sémiologie du stéréotype, Nathan, Paris, 1991 ; Jean-Louis Dufays, Stéréotype et lecture : essai sur la réception littéraire, Mardaga, Liège, 1994 ; Robert Frank, "Qu'estce qu'un stéréotype? ", in Jean-Noël Jeanneney (dir.), Une idée fausse est un fait vrai : Les stéréotypes nationaux en Europe, Odile Jacob, Paris, 2000, pp. 17-26.

12. Caroline Courbières, «La publicité à la lisière de l'art ou la propagation de modèles culturels entre l'habit et l'habiter», 2003, texte disponible [en ligne] : URL http://archivesic.ccsd.cnrs.fr 
référentiel ultra-mondain dans lequel le modèle de mode se métamorphose en modèle féminin signifié en tant que référent interne ${ }^{13}$. Le discours médiatique sur la mode met en œuvre un niveau méta-informationnel permettant de désigner le substrat culturel qui contextualise le double objet de message (la mode et la femme) et oriente son interprétation. L'analyse de ce stéréotypage particulier montre par ailleurs comment le figement constant ou, à l'inverse, les lentes évolutions qui affectent ces représentations du féminin participent à la fois de l'émergence ou du renforcement de stéréotypes nationaux ${ }^{14}$.

\section{LE MÉTADISCOURS DOCUMENTAIRE}

De la même manière que le discours médiatique sur la mode déploie sa dimension idéologique et impose ses propres représentations au-delà de sa sphère d'inscription, le langage documentaire forme un discours dont la substance du contenu déborde la visée informative neutralisée et neutralisante affichée. Tout langage documentaire invite de par son caractère normatif à être analysé en tant que discours sur ou métadiscours. Ce discours s'apparente à un " discours constituant » qui présente trois dimensions : un processus d'auto-instauration du discours, un " agencement d'éléments formant une totalité textuelle », et son établissement afin de servir "de norme et de garant aux comportements d'une collectivité ${ }^{15}$. Un langage documentaire se donne à lire dans une configuration signifiante spécifique qui se caractérise par l'utilisation des signes linguistiques, la structuration à l'aide de relations sémantiques - (en l'occurrence, des relations d'équivalence, des relations hiérarchiques et des relations associatives) et l'élaboration d'un cadre de classement conceptuel qui assigne aux unités documentaires leur place. Il se justifie $a$ priori d'après leur utilisation, c'est-à-dire en tant que systèmes de représentation, et a posteriori comme métadiscours de référence pour un groupe partageant une même pratique professionnelle. Sa légitimation se situe également dans sa fonction de représentation des savoirs.

En effet, ce type de langage artificiel - au sens, notamment, où il constitue un système d'expression construit à des fins documentaires - doit être en mesure de rendre compte du contenu d'objets documentaires à l'aide de ses unités : on parle d'« outils de représentation des connaissances » ou de "représentation des savoirs ». Le langage documentaire forme une grille langagière servant à condenser l'information afin de faciliter l'exploitation des objets indexés. Or, de par sa nature même, il élabore un contenu informationnel à travers lequel il se donne à interpréter : l'artifice documentaire définit le statut de ce langage

13. Caroline Courbières, «Le principe du stéréotypage médiatique de l'image féminine de mode : de La Belle au bois dormant à La possibilité d'une île ", in Henri Boyer (dir.), Stéréotypage, stéréotypes : fonctionnements ordinaires et mises en scène, tome 1, Média(tisation)s, actes du colloque international de Montpellier (21, 22 et 23 juin 2006, Université Montpellier III), L’Harmattan, Paris, 2007, pp. 99-108.

14. Caroline Courbières, «Des traces discursives aux figures féminines : la cristallisation médiatique de stéréotypes nationaux ", in Stéréotypes et prototypes nationaux en Europe, actes choisis du colloque international, (organisé par le Forum des langues européennes, Paris, 4-5 novembre 2005), Forum des langues européennes, Paris, 2007, pp. 130-139.

15. Dominique Maneau, Frédéric Cossuta, «L'analyse des discours constituants », Langages, 117, mars 1995, pp. 112-125. 
particulier, à la fois outil de représentation des connaissances et système langagier produisant ses propres représentations. Cette notion d'artifice permet de souligner la complexité de la représentation documentaire par laquelle se télescopent les niveaux linguistiques et extralinguistiques. Ce télescopage, saisissable dans la structure même du langage, se négocie dans et par l'interprétation, qui actualise les virtualités proposées ${ }^{16}$. Un langage documentaire résulte de la tension entre le dit documentaire (les connaissances devant être représentées) et le dire documentaire, c'est-à-dire le système élaboré à partir des unités sémantiques censées représenter le contenu informationnel des objets documentaires. Cette tension s'exprime dans le statut paradoxal du langage documentaire, à la fois calque des objets à représenter et système de construction de représentations langagières.

On peut dès lors convenir, d'une part, que l'analyse du langage documentaire en tant qu'outil vise davantage à déterminer sa fonction de désignation des connaissances à représenter. Cette analyse considère l'unité du langage documentaire comme une donnée disponible - parmi d'autres - pour désigner, à travers sa représentation conceptuelle, un objet référentiel (de la connaissance). L'analyse du langage en tant que discours documentaire, d'autre part, s'intéresse en premier lieu à son sens, résultant de la configuration signifiante constituée par des unités documentaires définies les unes par rapport aux autres par des relations sémantiques. C'est à partir de ce jeu combinatoire que peut être réalisé un parcours interprétatif qui permet de reconstruire le sens de chacune des unités. Cette seconde option s'attache au signe documentaire, dont la valeur dépend de sa situation au sein du système langagier. Appréhender le langage documentaire comme discours susceptible d'engendrer un processus de stéréotypage amène à se situer du côté du dire documentaire et implique d'approfondir sa matérialité en tant que système de signes.

Un langage documentaire peut ainsi se définir comme un système d'unités combinées entre elles selon des rapports différentiels : ses unités se manifestent sur l'axe syntagmatique selon des rapports combinatoires in praesentia (en l'occurrence réglés par les relations documentaires hiérarchiques), et entretiennent également des rapports associatifs in absentia sur l'axe paradigmatique avec d'autres unités non réalisées ou actualisées. Mais ce type de langage présente en outre la spécificité d'exprimer certains de ces rapports paradigmatiques à travers les relations d'équivalence et les relations associatives. Cette approche, d'inspiration saussurienne, permet in fine de dévoiler le mécanisme de stéréotypage documentaire articulé par les différentes relations sémantiques constitutives du langage documentaire.

\section{StÉrÉotYPAgE dOCUMENTAIRE}

Le stéréotypage documentaire est généré à la fois par les relations d'équivalence et par les relations hiérarchiques et associatives qui établissent le contexte de signification des unités langagières. Pour les langages documentaires linguistiques, le choix des unités et leur combinaison associative constituent le cœur des 16. François Rastier, Sémantique interprétative, $3^{\mathrm{e}}$ éd. mise à jour, PUF, Paris, 2009. 
opérations paradigmatiques. Cette sélection des unités se manifeste à travers la relation d'équivalence qui indique les unités à employer et les unités exclues, ce afin de réduire leur nombre. Par exemple, un langage documentaire posera la notion de genre humain comme équivalente à celles d'espèce humaine et d'homme, et choisira cette dernière unité pour la représenter. La relation d'équivalence renvoie à la notion de synonymie qui, en documentation, se démarque de celle définie en langue. En effet, si l'on ne peut pas parler de véritable relation d'identité (sémantique) entre des unités du plan du contenu - la synonymie ne pouvant être que partielle -, un langage documentaire de type thésaurus ou liste d'autorité matière pose le principe de synonymie afin de résoudre plusieurs cas sémantiques : on peut tout d'abord qualifier de synonymes des termes qui ont le même sens ou presque ; cette première " équivalence documentaire $" ~ "{ }^{17}$ ne peut se concevoir dans le cadre d'une théorie de la signification selon lequel toute unité signifiante est corrélée à un signifié unique. On peut ensuite instituer des quasi-synonymes, c'est-à-dire assimiler des termes spécifiques à un terme plus générique : le langage documentaire opère un écrasement de l'unité documentaire par l'instauration d'une relation syntagmatique, la relation d'équivalence s'apparentant alors à une relation de type hiérarchique. Enfin, la relation de synonymie documentaire peut se définir par son exact opposé en langue, à savoir, par une relation d'antonymie : dans ce cas, la relation d'équivalence regroupe des antonymes - (c'est-à-dire des contraires qui existent en contexte) - sous un terme unique.

La synonymie documentaire relève ainsi conjointement d'une théorie de la désignation (ou de la référence) - où la synonymie est envisageable - et d'une théorie de la signification - qui s'occupe de l'antonymie -, brouillant la saisie sémantique des unités documentaires : soit celles-ci sont amputées de leur signifié spécifique, renvoyant à un référent concret extralinguistique ; soit, au contraire, leur choix se fonde sur la concordance (quasi-synonymes hiérarchiques) ou l'opposition (quasi-synonymes antonymes) sémiques qu'elles entretiennent avec d'autres unités. La relation d'équivalence documentaire illustre dans sa fonctionnalité même toute l'artificialité de ces langages qui, en tant qu'outils de représentation des connaissances, relèvent des problèmes d'une sémantique référentielle et/ou formelle, s’intéressant aux rapports entre concept, référent et signe, et qui, en tant que discours documentaires, se prêtent à une approche sémio-communicationnelle.

On voit bien comment la tension dont résulte tout langage documentaire se résout ici dans la détermination du dit documentaire (les éléments informationnels à représenter) par le dire documentaire (les unités documentaires signifiantes). Il ne s'agit plus dès lors de parler de représentation des connaissances, mais - avant tout - de (re)construction de représentations langagières comme forme et substance du contenu linguistique documentaire. La condensation de l'information réalisée sur le plan paradigmatique pulvérise le statut sémantique des unités documentaires, non seulement en les projetant sur l'axe syntagmatique du langage, mais aussi en complexifiant leur point de référence. La réduction

17. Muriel Amar, Les fondements théoriques de l'indexation: Une approche linguistique, ADBS Éditions, Paris, 2000. 
sémique à la base de l'établissement des relations d'équivalence a pour corollaire l'éparpillement sémantique réalisé par les relations dites associatives. Ces relations peuvent exprimer différents liens que l'on peut classer selon l'appartenance des unités ainsi reliées, soit à des hiérarchies distinctes, soit à une même hiérarchie. Le détail de ces deux catégories montre comment les relations d'association peuvent en fait signifier des relations hiérarchiques ou des relations d'équivalence : la première catégorie permet en effet d'instaurer une relation partitive - substituant de fait une relation associative à une relation hiérarchique - ; le contenu de la seconde catégorie autorise pour sa part la substitution d'une relation associative à une relation d'équivalence. Au-delà de la complexité de leur nature, les relations associatives relèvent du processus de fragmentation documentaire qui distribue la signification d'une unité au travers d'autres unités.

\section{FigeMENT ET FRAGMENTATION}

Le phénomène de stéréotypage documentaire procède donc de la combinaison de deux caractéristiques intrinsèques au langage documentaire : le principe de figement documentaire et le processus de fragmentation documentaire. La première caractéristique relève de la définition même du langage documentaire conçu comme langage artificiel ; la seconde est consécutive à la manifestation de l'ordre paradigmatique sur l'axe syntagmatique.

La notion de figement est empruntée au domaine linguistique : le figement lexical, "caractéristique universelle des langues naturelles ${ }^{18}$, est le processus suivant lequel des unités lexicales distinctes se trouvent solidifiées dans la composition d'une nouvelle unité. Il se déroule donc sur le plan syntagmatique et possède un triple effet sémantique/lexical/syntaxique ${ }^{19}$. Ce processus crée des locutions relevant de la phraséologie et se rapproche de la notion de lexie complexe : l'éternel féminin, par exemple, fait partie des « composés complexes » qui témoignent d'une « rupture paradigmatique ${ }^{20}$. Le figement documentaire s'entend pour sa part au niveau de l'acception sémantique de la lexie simple ou composée - telle qu'elle apparaît comme unité choisie dans le langage documentaire -, et procède en deux temps distincts : il réside tout d'abord dans la formalisation d'un signifié par un signifiant unique puis se voit consolidé par l'imposition des relations hiérarchiques.

Le figement préalable découle de la raison d'être des langages documentaires, à savoir la transposition double du signifiant et du signifié comme spécificité de ces langages parmi les langages artificiels. Pour chaque unité documentaire, la forme de l'expression se voit dotée d'une forme du contenu unique selon le processus terminologique adopté par le langage documentaire. Ce figement préalable est redoublé par les relations d'équivalence qui manifestent les virtualités selon lesquelles une unité $a$ \{signifiant $1 /$ signifié 1$\}$ est substituable à une unité

18. Gaston Gross, « Degré de figement des noms composés », Langages, 23(90), 1988, pp. 57-72.

19. Gaston Gross, Les expressions figées en français : noms composés et autres locutions, Paris, Ophrys, 1996.

20. Gaston Gross, «Définition des noms composés dans un lexique-grammaire », Langue française, 87, 1990, pp. 84-90. 
$b$ \{signifiant 2/signifié 1 '\}. Ce faisant, le langage documentaire pré-actualise ses unités et leur impose un sens en fixant leur signification. Puis le figement se trouve renforcé sur l'axe syntagmatique par les relations hiérarchiques qui assignent à l'unité documentaire une place donnée dans la structure conceptuelle du langage. Le principe de figement réalise dès lors la première étape du phénomène de stéréotypage documentaire : la signification d'une unité se trouve stabilisée par les relations d'équivalence qui lui accordent son statut documentaire et par les relations qui la subordonnent à une (ou plusieurs) autre(s) unité(s) à l'intérieur d'une hiérarchie sémantique identifiée.

La fragmentation documentaire se définit par l'exposition fragmentée de l'ordre paradigmatique. L'utilisation de ce terme de fragmentation se rapproche de l'emploi qui en est fait pour désigner sur Internet la transformation de l'unité documentaire à travers les phénomènes de dilution et de décontextualisation ${ }^{21}$. La fragmentation dont il est question dans le cadre du langage documentaire se rapproche de ces niveaux de déconstruction puisque l'unité (au sens d'unité constitutive du langage documentaire) se voit disséminée dans différents domaines, perdant en quelque sorte son intégrité signifiante. En effet, l'exposition fragmentée de l'ordre paradigmatique se traduit à travers les relations associatives qui rendent possible la saisie du signifié d'une unité a sur le signifiant d'une unité $c$; cette récurrence du signifié projette l'unité documentaire dans différentes sphères sémantiques thématiques en réalisant des "liens interchamps » virtuels ${ }^{22}$. Le phénomène de stéréotypage se voit ainsi accentué à travers le processus de fragmentation selon lequel l'unité documentaire se trouve convoquée de manière privilégiée dans une autre classe sémantique par le biais de relation(s) associative(s) réitérée(s). Cette seconde étape du stéréotypage documentaire peut également donner lieu à un processus de défragmentation résultant de la fusion de relations associatives dans une unité documentaire.

Pour illustrer ce phénomène, on peut examiner l'une des facettes sous lesquelles le féminin se trouve inscrit dans le langage documentaire au cours de la seconde moitié du vingtième siècle. Si le discours documentaire ancre principalement le féminin dans le domaine médical ou physiologique, il élabore aussi la figure de la mère dans d'autres sphères en la dotant d'attributs exclusifs. La mère - à l'inverse de la figure du père - se voit par exemple spécifiée dans le thésaurus de l'Unesco ou dans Rameau ${ }^{23}$ comme mère au travail et mère célibataire; la maternité, potentialité exclusivement féminine, se trouve dès lors fusionnée avec la situation économique et avec le statut (non)conjugal. Ces deux unités opèrent ainsi un figement qui cristallise un processus de fragmentation au niveau même

21. Stéphane Chaudiron, Ihadjadene Madjid et Azzeddine Maredj, " La fragmentation et l'unité documentaire en question ", in Les sciences de l'information et de la communication : affirmation et pluralité, $16^{\mathrm{e}}$ Congrès de la SFSIC, 11-13 juin 2008, Compiègne. Texte disponible [en ligne] : adresse URL : http://www.sfsic.org/congres_2008/spip.php?article109

22. Bernard Pottier, «Le domaine de l'ethnolinguistique », Langages, 5(18), 1970, pp. 3-11.

23. Rameau (Répertoire d'autorité-matière encyclopédique alphabétique et unifié) est le langage documentaire élaboré depuis 1980 par la Bibliothèque nationale, en relation avec le Répertoire de vedettes-matière de l'Université Laval à Québec, lui-même traduit de la liste de vedettes-matière de la Bibliothèque du Congrès des États-Unis d'Amérique. 
des unités documentaires. La contextualisation du féminin ne se produit plus par l'appartenance d'une unité à deux domaines distincts, mais dans la production même d'une unité singulière : on assiste ici à un processus de défragmentation qui concentre deux notions auparavant reliées de manière associative. La mère se lit également par fragmentation, dans ces deux langages, en tant que femme figée dans le rôle de femme au foyer, l'assignation culturelle de la femme aux tâches domestiques s'exprimant dans le figement réalisé par le choix de la lexie composée. Cette caractéristique documentaire du féminin, associée à la notion de maternité, croise l'inscription de la femme en tant que travailleuse : la Classification décimale universelle et la Classification décimale de Dewey octroient aux femmes et/ou aux mères une place dans le domaine du travail : soit les femmes sont distinguées des mères, soit elles sont regroupées sous l'intitulé femmes mariées, mères travailleuses à l'issue d'un processus de fragmentation qui complexifie davantage la représentation du féminin.

\section{L'ARTIFICE DOCUMENTAIRE}

De manière plus générale, le souci de travailler ces champs associatifs réside dans l'idée saussurienne du terme comme " centre d'une constellation » : toute unité linguistique est susceptible de générer des associations sur le plan de son signifiant et de son signifié. De la même manière, toute unité documentaire peut être vue comme le centre d'une thématique qui, en l'occurrence, exprime les virtualités de l'unité en projetant l'axe paradigmatique sur l'axe syntagmatique (notamment par le biais des relations associatives). Cette thématique documentaire, saisissable à travers les thèmes, domaines ou classes documentaires générant les relations hiérarchiques, se construit également par les rapports paradigmatiques qui lient les unités entre elles avant même que celles-ci soient actualisées. L'artifice documentaire se révèle à réception par l'actualisation d'isotopies, entendues ici comme faits syntagmatiques de récurrence sémique.

La notion d'artifice documentaire renvoie certes à l'artificialité selon laquelle se définit traditionnellement le langage documentaire en tant que système descriptif d'objets documentaires, mais cherche surtout à caractériser la nature sémiotique propre à cette structure signifiante particulière. En effet, le caractère artificiel de tout langage documentaire affecte la nature de ses unités documentaires, que ce langage soit pris comme outil de représentation des connaissances ou considéré comme objet discursif à part entière. Dans le premier cas, ses unités documentaires représentent de simples données, perdant leur contenu informationnel social. En revanche, dans le second cas, elles constituent des représentations langagières relevant d'une catégorisation particulière, soumises à l'interprétation. L'artifice documentaire réside alors dans la manière dont s'opère la fonction sémiotique dans ce langage.

La structuration à la fois syntagmatique et paradigmatique du langage documentaire permet d'éclairer cet artifice et de mettre au jour le mécanisme de stéréotypage à l'œuvre en rendant saillants le principe de figement et le processus de fragmentation documentaires : le principe de figement documentaire assigne sa signification à une unité documentaire en la reliant, d'une part, à une (ou d'autres) unité(s) dite(s) équivalente(s) et, d'autre part, en la maintenant dans une 
hiérarchie conceptuelle fixe; le processus de fragmentation documentaire pulvérise la saisie d'une unité en l'associant à une (ou plusieurs) unité(s) relevant d'un autre domaine thématique.

L'examen de l'établissement des différentes relations qui sous-tendent l'architecture sémantique de ce système de signes spécifique montre ainsi comment se fabrique le contexte de signification des unités documentaires et comment s'opère leur cristallisation langagière.

Ce phénomène de stéréotypage, inhérent à la thématique documentaire instituée, balise les parcours interprétatifs possibles à réception. À production, il propage les " miettes lexicales » du discours documentaire, qui " représentent en même temps les lieux communs, c'est-à-dire les banalités de chacun des micro-univers du savoir $»^{24}$.

\section{CAROLINE COURBIÈRES}

